\title{
CORRESPONDENCE
}

\section{Is vinclozolin a reproductive hazard to} men?

Zober et al ${ }^{1}$ examined fertility, hormones, and offspring sex ratio of men exposed to the fungicide vinclozolin. These authors were inclined to dismiss the possibility that it has any deleterious effect on the male reproductive system. I should like to suggest in contrast, that its effects in two respects resemble those of the nematocide dibromochloropropane (DBCP), which lowers sperm count ${ }^{2}$ and is associated with a highly significantly lowered offspring sex ratio. ${ }^{3}$

Zober et $a l^{1}$ report that although the testosterone concentrations of exposed men were unchanged their follicle stimulating hormone (FSH) concentrations were significantly higher than those of controls $(P=$ 0.004). Similarly, increased FSH and unchanged testosterone were found in men exposed to DBCP. ${ }^{4}$ Increased gonadotrophin, or lowered testosterone concentrations, or both are characteristic of many illnesses in men. ${ }^{5}$

The offspring of men exposed to vinclozolin numbered 44 sons and 51 daughters. ${ }^{1}$ Compared with an expected ${ }^{6}$ Caucasian live birth sex ratio (proportion male) of 0.515 , these figures yield a $\chi^{2}$ of $1.05(P<0.15$, one way). So although not formally significant, they may be thought to be suggestive.

I suggest that further study of the possible effects of vinclozolin on the male reproductive system are merited. Its known similarities to an established hazard, DBCP, are disturbing. Especially interesting (and easy to gather) would be the sex ratios of offspring of men exposed to vinclozolin.

\section{WILLIAM H JAMES}

The Galton Laboratory, University College London, Wolfson House, 4 Stephenson Way, London NW1 $2 H E$

1 Zober A, Hoffmann G, Ott MG, Will W, Germann C, Van Ravenzwaay B. Study of the morbidity of personnel with potential the morbidity of personnel with potential 1995;52:233-241.

2 Whorton D, Krauss RM, Marshall S, Milby TH. Infertility in male pesticide workers. Lancet 1977;ii: $1259-61$.

3 Potashnik G, Yanai-Inbar I. Dibromochloropropane (DBCP): an 8-year re-evaluation of testicular function and reproductive performance. Fertil Steril 1987;47:317-23.

4 Whorton D, Milby TH, Krauss RM, Stubbs HA. Testicular function in DBCP-exposed pesticide workers. $f$ Occup Med 1979;21: 161-6.

5 Semple CG. Hormonal changes in nonendocrine disease. $B M \mathcal{F}$ 1986;293:1049-52.

6 James WH. The human sex ratio. Part I: a review of the literature. Hum Biol 1987;59: 721-52.

Authors reply-In his letter, James raises questions about two specific health outcome measures reported in our paper, namely, serum follicle stimulatory hormone (FSH) and sex of children born to fathers in the study group, and suggests that our findings for vinclozolin are similar to those reported by others for dibromochloropropane (DBCP), a known reproductive toxin in men.
As was pointed out by James, the mean value of the serum FSH measurements was significantly higher in the exposed than in the control group $(P=0.004$ both before and after exclusions based on known preexposure health conditions) and this would suggest a possible causal relation between exposure and outcome. However, for both luteinising hormone (LH) and FSH, nonsignificant but negative dose-response trends were found relative to current intensity as well as estimates of cumulative dose of vinclozolin exposure (see fig 4 of original paper for scatter plot of FSH $v$ current intensity measure). Also, the man in the exposed group whose FSH level of $14.0 \mathrm{mU} / \mathrm{ml}$ was at the upper limit of normal had unilateral atrophy only later found to have been present since childhood. All other readings among exposed men were within the reference range. From toxicological studies on vinclozolin and studies on other agents that block the testosterone receptor such as flutamide, the pattern of gonadotrophins at effective dose rates typically consists of increased testosterone, greatly increased LH, and marginally increased FSH concentrations. ${ }^{12}$ In the absence of dose-response and any abnormal FSH findings within the restricted exposed group and the lack of similarity between observed gonadotrophin patterns and what would be expected for an antiandrogenic agent, it is difficult to conclude that our findings are indicative of an effect related to vinclozolin.

Because the medical histories reported in table 3 of our paper did not distinguish between births occurring before and after first exposure, we reviewed the medical and work history data of all study group participants to find the number and sex of children born nine or more months after the date of the first exposure. Within this restricted observation period, there were 31 births, 13 male and 18 female, reported by $21 \mathrm{mem}$ bers of the study group. The number of births per family unit ranged from one to three. There were eight families with more male than female children born after exposure, 11 families with more female than male children and two families with one male and one female child born after exposure. These more detailed data are consistent with the trend noted by James, but are also consistent with a chance distribution of children by sex. Again available toxicological data for vinclozolin indicate that at toxicologically effective doses, it is not the sex ratio of offspring that is affected, but rather development in males.

A further issue raised by James was the resemblance between the effects of vinclozolin and those of DBCP on the basis of our FSH and sex ratio findings. From a toxicological viewpoint, there are few similarities between the two substances. DBCP has been shown to affect testicular tissue through direct genotoxic effects. ${ }^{3}$ Vinclozolin is not genotoxic and does not affect Sertoli cells directly, but rather acts by blocking the testosterone receptors. ${ }^{12}$ This mechanism of action is quite different rom that of DBCP and the anticipated pattern of gonadotrophin findings and their implications would be expected to be different as well.

In conclusion, we do concur with the suggestion that further systematic observation of people likely to have contact with vinclozolin is prudent and would be of scientific merit. However, we do not agree with the analogy drawn between vinclozolin and DBCP, which is not, in our opinion, supported by existing toxicological and epidemiological data.

\section{A ZOBER A G OTT \\ B VAN RAVENZWAAY}

1 van Ravenzwaay B. Discussion of prenatal and reproduction toxicity of Reg No 83258 (vinclo-
zolin). Data submission to US EPA. Washington: BASF Corporation MRID, Washington: BASF Con

2 JMPR. Report of the Joint Meeting of the FAO Panel of Experts on Pesticide Residues in Food and the Environment and the WHO Expert Group on Pesticide Residues. Food and agriculture organization of the United Nations. Geneva: WHO 1995:191-4.

$3 \mathrm{Lag} \mathrm{M}$, Soderlung EJ, Omichinski JG, Brunborg G, Holme JA, Dahl JE, et al. Effect of bromine and chlorine positioning in the induction of renal and testicular toxicity by halogenated propanes. Chemical Research in halogenated propanes. Chem
Toxicology 1991;4:528-34.

Modifiers of non-specific symptoms in occupational and environmental syndromes

Editor-In a thorough review Spurgeon et $a l^{1}$ concluded that various occupational or environmental factors may lead to an increase of non-specific symptoms such as headache, tiredness, eye irritation, and backache, when they are (rightly or wrongly) perceived as health hazards. Dissatisfaction with specific aspects of work may stimulate the occurrence of a similar pattern of health complaints. The authors discussed the role of individual and social factors in the perception of health and the tendency to report symptoms. Most probably some people are more sensitive to the psychosocial factors mentioned than others

Spurgeon et al did not pay attention to one point. If anxiety and dissatisfaction affect the perception of health, there might be a comparable effect on the perception of environmental factors. If so, negative or anxious feelings may also intensify the reporting of complaints about aspects of work in a non-specific way.

Many studies in the field of occupational epidemiology are (at least partly) based on self reported health or work related exposures. It is obvious that the tendencies mentioned may lead to biased results. Any particular problem that crops up in a work situation may generate anxiety or dissatisfaction, which in turn stimulates the tendency to report non-specific symptoms and complaints. Assuming that this tendency is stronger in some people than others, spurious associations between exposure and effect can be expected. This is an additional reason to explore how psychosocial factors may colour the reporting of complaints. ${ }^{1}$ Two topics should therefore be added to the recommendations for further research as formulated by Spurgeon et al.

(1) Empirical evidence should be searched for our hypothesis that feelings of anxiety or dissatisfaction may lead to increased complaints about (probably nonspecific) aspects of work. Research done on 